\title{
Group Duality and the Kubo-Martin-Schwinger Condition
}

D. Kastler ${ }^{1}$ and M. Takesaki ${ }^{2 \star \star \star}$

Centre de Physique Théorique du CNRS II, Marseille-Luminy, Marseille, France

1 Département de Physique, UER Scientifique de Marseille-Luminy, Marseille, France

2 Département de Mathématiques, UER Scientifique de Marseille-Luminy, Marseille, France

\begin{abstract}
We consider clustering $G$-invariant states of a $C^{*}$-algebra $\mathfrak{A}$ endowed with an action of a locally compact abelian group $G$. Denoting as usual by $F_{A B}, G_{A B}$, the corresponding two-point functions, we give criteria for the fulfillment of the KMS condition (w.r.t. some one-parameter subgroup of $G$ ) based upon the existence of a closable map $T$ such that $T F_{A B}=G_{A B}$ for all $A, B \in \mathfrak{A}$. Closability is either in $L^{\infty}(G), B(G)$, or $\mathscr{C}_{\infty}(G)$, according to clustering assumptions. Our criteria originate from the combination of duality results for the group $G$ (phrased in terms of functions systems), with density results for the two-point functions.
\end{abstract}

\section{Introduction}

The so-called Kubo-Martin-Schwinger (KMS) condition plays an important role both in physics, where it is the modern expression of the "Gibbs structure" (independant of the thermodynamic limit) [1] and in the theory of von Neumann algebras where separating normal states possess this property w.r.t. their "modular automorphism groups" [2]. With $\mathfrak{A}$ a $C^{*}$-algebra and $t \rightarrow \alpha_{t}$ a one-parameter automorphism group of $\mathfrak{A}$, a state $\omega$ is called $\beta$-KMS for $\alpha$ whenever, to each pair $A, B \in \mathfrak{A}$, there is a function $f$ of the complex variable, continuous and bounded in the strip $0 \leqq \operatorname{Im} z \leqq \beta$, holomorphic in its interior, with boundary values

$$
\left\{\begin{array}{l}
F_{A B}(t)=\omega\left(B \alpha_{t}(A)\right)=f(t) \\
G_{A B}(t)=\omega\left(a_{t}(A) B\right)=f(\beta t+i), \quad t \in \mathbb{R} .
\end{array}\right.
$$

This condition can alternatively be stated as follows in terms of Fourier transforms (tempered distributions)

$$
\hat{F}_{A B}(p)=e^{-\beta p} \hat{G}_{A B}(p), \quad p \in \mathbb{R} .
$$

* Permanent address: Department of Mathematics, UCLA, Hilgard Avenue, Los Angeles, California, USA

$\star \star$ Supported in part by the National Science Foundation 
It is clear from these relations that the KMS condition implies the existence of a well-behaved map $F_{A B} \rightarrow G_{A B}$. Our purpose in this paper is to study the KMS condition in relation with this map and to see wether the existence of an appropriate map entails the condition. In fact we will consider the case of general continuous abelian automorphism groups and of KMS states w.r.t. a continuous one-parameter subgroup: inferring the KMS property for such a subgroup is then a procedure of the same nature as that of reconstructing a group from a dual object and this explains how our question leads us to duality. We restricted ourselves here to the abelian group case but it would be interesting, both physically and mathematically, to investigate in this sense the non abelian automorphism groups (in fact our study of the chemical potential in collaboration with Araki and Haag [3] pertains to a non abelian case - and Tatsuuma also brought a contribution in that direction [4]).

In fact we hope that our paper will provide part of the equipment for developing further algebraic statistical mechanics, and we are thinking in particular of the two following directions: on the one hand the derivation of the KMS structure from a combination of locality (here represented by asymptotic abelianness) and a quantum dynamical ergodicity (non splittability of the dynamical system) with which we hope that the existence of the map $F_{A B} \rightarrow G_{A B}$ is connected in some way. The other direction concerns wider automorphism groups, moving frames, symmetry breaking, etc.

Section 2 of this paper is purely mathematical. It provides a fourfold duality result (Corollary 2.7), whose proof is cast in the language of abstract Banach algebras in order to unify the approach for the different cases.

Section 3 is independent from Sect. 2 and deals with invariant states of dynamical systems. It provides density results (from physically meaningful assumptions) which ensure a sufficient size of the domain of the map $F_{A B} \rightarrow G_{A B}$.

Section 4 combines the results of the two preceding sections to develop criteria for the validity of KMS based on the existence of an appropriate map $F_{A B} \rightarrow G_{A B}$. We conclude with a short proof of an already known physical result as an illustration.

\section{Positive Characters and Duality ${ }^{1}$}

The classical Pontrjagin-Kampen duality theorem tells us how one can identify a given object with an element of a preassigned locally compact abelian group $G^{2}$. Namely, $G$ is exactly identified with the set of all continuous unitary characters of the dual group $\hat{G}$ of $G$. In this section, we shall study a method of identifying a oneparameter subgroup of $G$.

Suppose that $\{g(t) ; t \in R\}$ is a continuous one-parameter subgroup of $G$, i.e. a continuous homomorphism of the additive group $\mathbb{R}$ into $G$. The classical duality theorem entails at once that there exist a continuous homomorphism $\beta$ : $p \in \hat{G} \mapsto \beta(p) \in \mathbb{R}$ such that

$$
\langle g(t), p\rangle=\exp (i t \beta(p)), \quad t \in \mathbb{R}, p \in \hat{G},
$$

1 A general reference for this chapter is [5]

2 We shall use the additive notation for the group operations in $G$ and $\hat{G}$ 
where $\langle g, p\rangle$ means the value of $p \in \hat{G}$ at $g \in G$. We then consider a positive character $\chi_{\beta}$ of $\hat{G}$ given by

$$
\chi_{\beta}(p)=\exp \beta(p), \quad p \in \hat{G} .
$$

Thus, a continuous one-parameter subgroup $g(t)$ gives rise to a continuous positive character on $\hat{G}$. Conversely, we have the following:

Proposition 2.1. If $\chi$ is a positive continuous character of $\hat{G}$, then there exists a continuous one-parameter subgroup $\left\{g_{\chi}(t)\right\}$ of $G$ such that

$$
\chi(p)^{i t}=\left\langle g_{\chi}(t), p\right\rangle, \quad t \in \mathbb{R}, p \in \hat{G} .
$$

We leave the proof to the reader. It is a straightforward application of the duality theorem.

Thus, one can identify continuous one-parameter subgroups of $G$ with continuous positive characters on $\hat{G}$. If we drop the positivity assumption from a continuous character $\chi$ on $\hat{G}$, then we get a continuous one-parameter subgroup $\left\{g_{|\chi|}(t)\right\}$ of $G$ corresponding to the absolute value of $\chi$, and a single element $g_{0} \in G$ corresponding to the phase of $\chi$.

We shall then rephrase this characterization of a continuous one-parameter subgroup in terms of function systems over $G$, not directly involving $\hat{G}$. To this end, we consider the involutive Banach algebra $M(\hat{G})$ of finite Radon measures on $\hat{G}$, where the algebraic structure in $M(\hat{G})$ is defined as follows

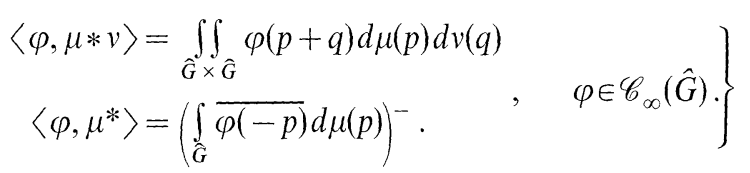

We denote here by $\mathscr{C}_{\infty}(\hat{G})$ the $C^{*}$-algebra of all continuous functions on $\hat{G}$ vanishing at infinity. For each $\mu \in M(\hat{G})$, we consider its inverse Fourier transform $\hat{\mathscr{F}} \mu$ defined by:

$$
(\hat{\mathscr{F}} \mu)(s)=\int_{\hat{G}}\langle s, p\rangle d \mu(p), \quad s \in G^{3} .
$$

It is known that $\hat{\mathscr{F}}$ is a $*$-isomorphism of $M(\hat{G})$ into the $C^{*}$-algebra $\mathscr{C}_{b}(G)$ of bounded continuous functions on $G$. We denote by $B(G)$ the range of $\hat{\mathscr{F}}$, and call it the Fourier-Stieljes algebra on $G$.

Suppose that we have a continuous positive character $\chi$ on $\hat{G}$ corresponding to a one-parameter subgroup $\{g(t) ; t \in \mathbb{R}\}$ of $G$. Set

$$
\begin{aligned}
& M_{\chi}=\{\mu \in M(\hat{G}) ; \chi \mu \in M(\hat{G})\} ; \\
& \mathscr{A}_{\chi}=\hat{\mathscr{F}}\left(M_{\chi}\right) .
\end{aligned}
$$

Define a linear map $T_{\chi}$ of $\mathscr{A}_{\chi}$ into $B(G)$ by:

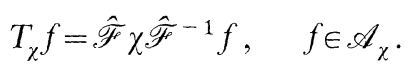

3 The Fourier transform $\mathscr{F}$ on $\hat{G}$ is defined by:

$$
(\mathscr{F} \mu)(s)=\int_{\hat{G}} \overline{\langle s, p\rangle} d \mu(p)=(\hat{\mathscr{F}} \mu)(-s), \quad \mu \in M(\hat{G}), s \in G
$$


Proposition 2.2. Under the above assumptions and notations, we have the following:

(i) $T_{\chi}$ is multiplicative and preserves positive definiteness;

(ii) $T_{\chi}$ extends to a multiplicative closed linear operator $T$ on $L^{\infty}(G)$, the closedness of $T$ refering to the $\sigma\left(L^{\infty}(G), L^{1}(G)\right)$-topology ${ }^{4}$;

(iii) for every $f$ in the domain $\mathscr{D}(T)$ of $T$ and every $s \in G$, there exists a bounded holomorphic function $F$ on the strip, $-1 \leqq \operatorname{Im} z \leqq 0$, such that

$$
\left.\begin{array}{rl}
F(t) & =f(g(t)+s) \\
F(t-i) & =(T f)(g(t)+s)
\end{array}\right\}
$$

for almost every $t \in \mathbb{R}$;

(iv) T commutes with translations.

Proof. Except possibly for (iii), it is straightforward to check the proposition.

Suppose $f=\hat{\mathscr{F}} \mu$ with $\mu \in M_{\chi}$. We have

$$
f(s+g(t))=\int_{\hat{G}}\langle s, p\rangle \chi(p)^{i t} d \mu(p), \quad s \in G, t \in \mathbb{R} .
$$

For a $z \in \mathbb{C}$ with $-1 \leqq \operatorname{Im} z \leqq 0$, set

$$
F(z)=\int_{\hat{G}}\langle s, p\rangle \chi(p)^{i z} d \mu(p) .
$$

Since $\chi$ is integrable with respect to the absolute value $|\mu|$ of $\mu, F$ is bounded and holomorphic inside the strip and we have

$$
F(t)=f(g(t)+s) \text { and } F(t-i)=\left(T_{\chi} f\right)(g(t)+s) .
$$

We next consider a strongly continuous one-parameter group $\left\{T_{t}^{*}\right\}$ of isometries on $L^{1}(G)$ defined by:

$$
\left(T_{t}^{*} f\right)(s)=f(s-g(t)), \quad f \in L^{1}(G), s \in G,
$$

and its adjoint group $\left\{T_{t}\right\}$ on $L^{\infty}(G)$ defined by:

$$
\left(T_{t} f\right)(s)=f(s+g(t)), \quad f \in L^{\infty}(G) .
$$

We also consider the set $H^{\infty}(D)$ of bounded functions on the strip $D=\{z \in \mathbb{C}$; $-1 \leqq \operatorname{Im} z \leqq 0\}$, holomorphic inside $D$. Let $\mathscr{D}$ be the set of all $f \in L^{\infty}(G)$ such that for every $g \in L^{1}(G)$, the function $t \in \mathbb{R} \mapsto\left\langle T_{t} f, g\right\rangle \in \mathbb{C}$ extends to an element $F_{g}$ of $H^{\infty}(D)$ with $\left\|F_{g}\right\|_{\infty} \leqq K_{f}\|g\|_{1}$ for some constant $K_{f} \geqq 0$. Clearly we have $\mathscr{A} \subset \mathscr{D}$. For each $z \in D$ and $f \in \mathscr{D}$, there exists $f_{z} \in L^{\infty}(G)$ such that

$$
\left\langle f_{z}, g\right\rangle=F_{g}(z), \quad g \in L^{1}(G) .
$$

If we set $T_{z} f=f_{z}$ for each $f \in \mathscr{D}$ and $z \in D$, then we have

$$
T_{\chi} f=T_{-i} f, \quad f \in \mathscr{A} .
$$

We shall show that $T_{-i}$ is closed. Let 05 be the graph of $T_{-i}$ in $L^{\infty}(G) \oplus L^{\infty}(G)$. We must show that $\left(5\right.$ is weak*-closed. Since $L^{\infty}(G) \oplus L^{\infty}(G)=\left(L^{1}(G) \oplus L^{1}(G)\right)^{*}$, we

$4 L^{\infty}(G)$ and $L^{1}(G)$ refer to a Haar measure on $G$, while $L^{\infty}(\hat{G})$ and $L^{1}(\hat{G})$ refer to the Plancherel measure on $\hat{G}$ 
need only to prove that the unit ball of $\left(\mathfrak{5}\right.$ is weak*-closed. Let $f_{n} \oplus T_{-i} f_{n} \in \mathbb{6}$ with $\left\|f_{n}\right\|_{\infty},\left\|T_{-i} f_{n}\right\|_{\infty} \leqq 1$, converge to $f \oplus g$ in the weak* topology. If $h \in L^{1}(G)$, the functions : $z \in D \mapsto\left\langle T_{z} f_{n}, h\right\rangle \in \mathbb{C}$ belong to $H^{\infty}(D)$ and are bounded uniformly by 1 . Since $\left\{T_{t}\right\}$ is weak* continuous, we have

$$
\left\langle T_{t} f, h\right\rangle=\lim _{n=\infty}\left\langle T_{t} f_{n}, h\right\rangle \text { and }\left\langle T_{t} g, h\right\rangle=\lim _{n=\infty}\left\langle T_{t-i} f_{n}, h\right\rangle \text {. }
$$

By the maximum modulus principle, the function: $t \mapsto\left\langle T_{t} f, h\right\rangle \in \mathbb{C}$ extends to an element $F_{h}$ of $H^{\infty}(D)$ and $\left\|F_{h}\right\|_{\infty} \leqq\|h\|_{1}$. Thus $f$ belongs to $\mathscr{D}$ and $g=T_{-i} f$. We set $T=T_{-i}$. By construction the analyticity requirement for $T$ follows.

Let $\mathscr{A}$ and $\mathscr{B}$ be two involutive semi-simple Banach algebras such that $\mathscr{A}$ is the dual space of $\mathscr{B}$ as a Banach space. Suppose that

(i) there exists a $*$-isomorphism $\varpi$ of $\mathscr{A}$ into $L^{\infty}(G)$ such that $\varpi$ is weak* continuous and the transpose map $\varpi_{*}$ is also an isomorphism of the convolution algebra $L^{1}(G)$ into $\mathscr{B}$;

(ii) there exists a homomorphism $\varrho$ of $G$ into $\operatorname{Aut}(\mathscr{A})$ such that each $\varrho_{s}$ is a weak* continuous isometry of $\mathscr{A}$ and its adjoint $\varrho_{s}^{*}$ gives rise to a strongly continuous representation of $G$ on the Banach space $\mathscr{B}$; by:

(iii) $\varpi$ intertwines the action $\varrho$ of $G$ on $\mathscr{A}$ and the action $\lambda$ of $G$ on $L^{\infty}(G)$ given

$\left(\lambda_{s} f\right)(r)=f(s+r), \quad f \in L^{\infty}(G), r, s \in G$.

(iv) The spectrum of $\mathscr{B}$ is the dual group $\hat{G}$ and the composition of the Gelfand representation $\hat{\varpi}$ of $\mathscr{B}$ and $\varpi_{*}$ coïncides with the Fourier transform $\mathscr{F}$ on $L^{\prime}(G)$.

In applications, we consider the following two examples of $\{\mathscr{A}, \mathscr{B}, \varrho, \varpi, \hat{\varpi}\}$ :

Example 2.3. Let $\mathscr{A}=M(\hat{G})$, the convolution algebra of finite measures on $G$ and $\mathscr{B}=\mathscr{C}_{\infty}(\hat{G})$. We define $\varpi, \varrho$, and $\hat{\varpi}$ as follows :

$$
\left.\begin{array}{rlrl}
\varpi(\mu)(s) & =\int_{\hat{G}}\langle s, p\rangle d \mu(p) & & \\
d\left(\varrho_{s} \mu\right)(p) & =\langle s, p\rangle d \mu(p), & & \mu \in M(\hat{G}), s \in G, p \in \hat{G}, \\
\hat{\omega}(\varphi) & =\varphi, & & \varphi \in \mathscr{C}_{\infty}(\hat{G})
\end{array}\right\}
$$

It is straightforward to check the above postulates. In this case, we have $\varpi(\mathscr{A})=B(G)$, the Fourier-Stieljes algebra on $G$.

Example 2.4. Let $\mathscr{A}=L^{\infty}(G)$ and $\mathscr{B}=L^{1}(G)$. We define $\varpi, \varrho$, and $\hat{\varpi}$ as follows:

$$
\left.\begin{array}{rlrl}
\varpi(f) & =f, & & f \in L^{\infty}(G) ; \\
\varrho_{s} & =\lambda_{s}, & & s \in G ; \\
\hat{\varpi}(f)(p) & =\int_{G} \overline{\langle s, p\rangle} f(s) d s, & & f \in L^{1}(G), p \in \hat{G} .
\end{array}\right\}
$$

It is also straightforward to check the above requirements. In this case, we have $\hat{\varpi}(\mathscr{B})=A(\hat{G})$, the Fourier algebra over $\hat{G}$.

Theorem 2.5. Suppose that $\{\mathscr{A}, \mathscr{B}, \varpi, \varrho, \hat{\varpi}\}$ satisfy the above postulates (i) through (iv). Let $\mathscr{D}$ be a weak*-dense subalgebra of $\mathscr{A}$ globally invariant under the action $\varrho$ of G. If $T$ is a weak*-closed homomorphism of $\mathscr{D}$ into $\mathscr{A}$ commuting with $\varrho$, there exist a 
$s_{0} \in G$ and a continuous one-parameter subgroup $\{g(t) ; t \in \mathbb{R}\}$ of $G$ such that for each $a \in \mathscr{D}$ and $s \in G$ there exists $F \in H^{\infty}(D)$ with boundary values:

$$
\left.\begin{array}{rl}
F(t) & =\varpi(a)(g(t)+s) \\
F(t-i) & =\varpi(T a)\left(s+s_{0}+g(t)\right)
\end{array}\right\}
$$

for almost every $t \in \mathbb{R}$.

For the proof, we need the following elementary lemma, which is more or less known. We include the proof for the sake of completeness.

Lemma 2.6. Let $\mathbb{A}$ be a regular semi-simple abelian Banach algebra with spectrum $\Omega^{5}$. Then the ideal $\mathscr{K}_{\mathbb{A}}=\{f \in \mathbb{A}: \hat{f}$ has a compact support in $\Omega\}$, where $\hat{f}$ denotes the Gelfand representation of $f$, is smallest among all dense ideals of $\mathbb{A}$.

Proof. Let $\mathscr{I}$ be a dense ideal of $\mathbb{A}$, and $K$ a compact subset of $\Omega$. We shall show that $f \in \mathscr{I}$ whenever supp $\hat{f} \subset K$. Let $\mathscr{I}_{K}=\{f \in \mathbb{A} ; \hat{f}=0$ on $K\}$. Then $\mathscr{I}_{K}$ is a closed ideal of $\mathbb{A}$. Consider $\mathbb{A}_{K}=\mathbb{A} / \mathscr{I}_{K}$ and the canonical map $\pi_{K}: \mathbb{A} \rightarrow \mathbb{A}_{K}$. The regularity of $\mathbb{A}$ implies that the spectrum of $\mathbb{A}_{K}$ is canonically identified with $K$. Then the compactness of $K$ entails that $\mathbb{A}_{K}$ is unital. The image $\pi_{K}(\mathscr{I})$ of $\mathscr{I}$ is a dense ideal of the unital algebra $\mathbb{A}_{K}$, so that $\pi_{K}(\mathscr{I})=\mathbb{A}_{K}$. Hence $\mathscr{I}$ contains an element $h \in \mathbb{A}$ such that $\hat{h}=1$ on $K$. Hence $h f=f \in \mathscr{I}$ whenever supp $f \subset K$. Hence $\mathscr{K}_{\mathbb{A}} \subset \mathscr{I}$.

Proof of Theorem 2.5. We consider the graph $\mathbf{6}$ of $T$ in $\mathscr{A} \oplus \mathscr{A}$. The weak*closedness of $T$ yields that $\mathfrak{5}$ is closed under the weak* topology in $\mathscr{A} \oplus \mathscr{A}$ determined by $\mathscr{B} \oplus \mathscr{B}$. Since $T$ and $\varrho$ commute, $\mathfrak{G}$ is invariant under the action $\tilde{\varrho}=\varrho \oplus \varrho$ of $G$. Hence $\varrho$ gives rise to an action of $L^{1}(G)$ on $\mathbb{5}$, denoted also by $\varrho$ : this means that the action $\varrho_{f}, f \in L^{1}(G)$, defined by

$$
\varrho_{f}=\int_{G} f(s) \varrho_{s} d s, \quad f \in L^{1}(G)
$$

commutes with $T$, i.e. $\varrho_{f}(\mathscr{D}) \subset \mathscr{D}$ and $T \circ \varrho_{f}=\varrho_{f} \circ T$.

Let $\mathscr{D}^{*}$ denote the definition domain of the adjoint operator $T^{*}$ in $\mathscr{B}$, it follows that $\mathscr{D}^{*}$ is invariant under $\varrho_{f}^{*}, f \in L^{1}(G)$, and $T^{*} \circ \varrho_{f}^{*}=\varrho_{f}^{*} \circ T^{*}$. We next claim that

$$
\varrho_{f}^{*}(b)=\varpi_{*}(f) b, \quad f \in L^{1}(G), b \in \mathscr{B} .
$$

For all $a \in \mathscr{A}$ and $f, g \in L^{1}(G)$, we have

$$
\begin{aligned}
\left\langle a, \varrho_{f}^{*} \varpi_{*}(g)\right\rangle & =\left\langle\varrho_{f}(a), \varpi_{*}(g)\right\rangle \\
& =\int_{G}\left\langle\varrho_{s}(a), \varpi_{*}(g)\right\rangle f(s) d s=\int_{G}\left\langle\varpi\left(\varrho_{s}(a)\right), g\right\rangle f(s) d s \\
& =\int_{G}\left\langle\lambda_{s} \varpi(a), g\right\rangle f(s) d s=\iint_{G \times G} \varpi(a)(r+s) g(r) f(s) d r d s \\
& =\int_{G \times G} \varpi(a)(r) g(r-s) f(s) d r d s=\langle\varpi(a), f * g\rangle .
\end{aligned}
$$

Thus $\varrho_{f}^{*}\left(\varpi_{*}(g)\right)=\varpi_{*}(f) \varpi_{*}(g)$. Since $L^{1}(G)$ separates $\varpi(\mathscr{A}), \varpi_{*}\left(L^{1}(G)\right)$ is dense in $\mathscr{B}$; thus we get (2.22).

5 A semi-simple abelian Banach algebra is said to be regular if the hull-kernel topology coinncides with the weak* topology on the spectrum 
Therefore $\mathscr{D}^{*}$ is invariant under the multiplication by $\varpi_{*}\left(L^{1}(G)\right)$. For any $b \in \mathscr{D}^{*}$ and $c \in \mathscr{B}$, there exists a sequence $f_{n} \in L^{1}(G)$ such that $\lim _{n=\infty}\left\|\varpi_{*}\left(f_{n}\right)-c\right\|=0$, and we have $b c=\lim _{n=\infty} b \varpi_{*}\left(f_{n}\right)$ and

$$
T^{*}(b) c=\lim _{n=\infty} T^{*}(b) \varpi_{*}\left(f_{n}\right)=\lim _{n=\infty} T^{*}\left(b \varpi_{*}\left(f_{n}\right)\right) .
$$

Hence $b c \in \mathscr{D}^{*}$ and $T^{*}(b c)=T^{*}(b) c$. Therefore $\mathscr{D}^{*}$ is an ideal of $\mathscr{B}$. Furthermore $\mathscr{D}^{*}$ is dense. Since $\hat{\varpi} \circ \varpi_{*}$ is the Fourier transform of $L^{1}(G), \hat{\varpi}(\mathscr{B})$ contains $A(\hat{G})$, which means that $\mathscr{B}$ is regular. Thus, Lemma 2.6 entails that $\hat{\omega}\left(\mathscr{D}^{*}\right)$ contains $\hat{\varpi}(\mathscr{B}) \cap \mathscr{K}(\hat{G})$, in particular $\hat{\varpi}\left(\mathscr{D}^{*}\right) \supset A(\hat{G}) \cap \mathscr{K}(\hat{G})$.

We define an operator $\hat{T}$ on $\hat{\bar{\omega}}\left(\mathscr{D}^{*}\right)$ by

$$
\hat{T}=\hat{\varpi} \circ T^{*} \circ \hat{\varpi}^{-1} \text {. }
$$

We then have

$$
\hat{T}(\varphi \psi)=\hat{T}(\varphi) \psi, \quad \varphi \in \hat{\varpi}\left(\mathscr{D}^{*}\right), \psi \in \hat{\varpi}(\mathscr{B}) .
$$

Hence we have, for all $\varphi, \psi \in \hat{\bar{t}}\left(\mathscr{D}^{*}\right)$,

$$
\varphi \hat{T}(\psi)=\hat{T}(\varphi \psi)=\hat{T}(\varphi) \psi,
$$

which means that there exists a continuous function $\chi$ on $\hat{G}$ such that

$$
\hat{T}(\varphi)=\chi \varphi, \quad \varphi \in \hat{w}\left(\mathscr{D}^{*}\right) .
$$

Noticing that $\hat{\varpi}$ is a norm decreasing map of $\mathscr{B}$ into $\mathscr{C}_{\infty}(\hat{G})$, we have a dual map $\hat{\varpi}^{*}$ from $M(\hat{G})$ into $\mathscr{A}$. For each $f \in L^{1}(G)$ and $\mu \in M(\hat{G})$, we have

$$
\langle\mathscr{F} f, \mu\rangle=\left\langle\hat{\varpi} \circ \varpi^{*}(f), \mu\right\rangle=\left\langle\hat{\varpi}^{*}(\mu), \varpi^{*}(f)\right\rangle=\left\langle\varpi \circ \hat{\varpi}^{*}(\mu), f\right\rangle
$$

so that $\varpi \circ \hat{\varpi}^{*}(\mu)$ must be the Fourier transform $\mathscr{F}(\mu)$ of $\mu$. Since $\varpi$ is multiplicative on $\mathscr{A}, \hat{\varpi}^{*}$ maps the convolution product in $M(\hat{G})$ into the product defined in $\mathscr{A}$. Suppose that $\mu \in M(\hat{G})$ has the property that $\chi$ is integrable with respect to $|\mu|$. For every $b \in \mathscr{D}^{*}$, we have

$$
\begin{aligned}
\left\langle\hat{\omega}\left(T^{*} b\right), \mu\right\rangle & =\langle\hat{T} \hat{\varpi}(b), \mu\rangle=\int \hat{\omega}(b)(p) \chi(p) d \mu(p) \\
& =\langle\hat{\omega}(b), \chi \mu\rangle
\end{aligned}
$$

so that $\hat{\omega}^{*}(\mu)$ belongs to the domain $\mathscr{D}$ of $T$ and

$$
T \hat{\omega}^{*}(\mu)=\hat{\omega}^{*}(\chi \mu)
$$

for every $\mu \in M(\hat{G})$ with $\chi \mu \in M(\hat{G})$. The multiplicativity of $T$ together with that of $\hat{\varpi}^{*}$ entail that

$$
\chi(\mu * v)=(\chi \mu) *(\chi v)
$$

for all $\mu, v \in M(\hat{G})$ with $\chi \mu$ and $\chi v$ finite. Thus $\chi$ must be a continuous character of $\hat{G}$. Therefore, there exists an $s_{0} \in G$ such that

$$
\chi(p)=\left\langle s_{0}, p\right\rangle \exp \beta(p), \quad \hat{p} \in \hat{G},
$$


with $\beta(p)=\log |\chi(p)|$. Thus we obtain a continuous one-parameter subgroup $\{g(t)$; $t \in \mathbb{R}\}$ such that

$$
\langle g(t), p\rangle=e^{i t \beta(p)}, \quad p \in \hat{G} .
$$

Set $T_{t}=\varrho_{g(t)}, t \in \mathbb{R}$. We have a homomorphism $T: t \in \mathbb{R} \mapsto T_{t} \in \operatorname{Aut}(\mathscr{A})$ such that $\left\{T_{t}^{*}\right.$; $t \in \mathbb{R}\}$ is a strongly continuous one-parameter group of isometries of $\mathscr{B}$. Let $\mathscr{D}_{0}$ be the set of all those $a \in \mathscr{A}$ such that for every $b \in \mathscr{B}$, the function $t \in \mathbb{R} \mapsto\left\langle T_{t}(a), b\right\rangle \in \mathbb{C}$ extends to an element $F_{b}$ of $H^{\infty}(D)$ such that

$$
\left\|F_{b}\right\|_{\infty} \leqq k_{a}\|b\|
$$

with a constant $k_{a}>0$ independent of $b$. As in the proof of Proposition 2.1, we define a closed operator $T_{-i}$ with domain $\mathscr{D}_{0}$. We claim that $\varrho_{s_{0}}{ }^{\circ} T_{-i}$ extends $T$.

To prove the claim, we shall first show that if $\chi \hat{\omega}(b)=\hat{w}(c)$ with $b, c \in \mathscr{B}$ then $b \in \mathscr{D}^{*}$ and $T^{*} b=c$. For every $x \in \mathscr{D}^{*}$, we have

$$
\hat{\varpi}\left(T^{*} b x\right)=\chi \hat{\omega}(b x)=\chi \hat{\omega}(b) \hat{\omega}(x)=\hat{\omega}(c) \hat{\omega}(x)=\hat{\omega}(c x)
$$

so that

$$
T^{*} b x=c x, \quad x \in \mathscr{D}^{*} .
$$

By (2.22), $\hat{\oplus}^{-1}(\mathscr{K}(\hat{G}) \cap A(\hat{G}))$ contains an approximate identity $\left\{x_{n}\right\}$, so that we have

$$
b=\lim b x_{n} \text { and } \lim T^{*}\left(b x_{n}\right)=\lim c x_{n}=c .
$$

Thus $b \in \mathscr{D}^{*}$ and $T^{*} b=c$. From this, it follows that the image $\mathscr{D}_{1}$ of $\{\mu \in M(\hat{G})$; $\chi \mu \in M(\hat{G})\}$ under $\hat{\varpi}^{*}$ is a core of $T$. As in the proof of Proposition 2.1, we observe that

$$
\varrho_{s_{0}} \circ T_{-i} \hat{\omega}(\mu)=\hat{\varpi} *(\chi \mu)
$$

if $\mu$ and $\chi \mu$ belong to $M(\hat{G})$. Thus $T$ and $\varrho_{s_{0}} \circ T_{-i}$ coïncide on $\mathscr{D}{ }_{1}$. We completed the proof.

Corollary 2.7. (i) Let $\mathscr{D}$ be a weak*-(resp. norm-) dense subalgebra of $L^{\infty}(G)$ (resp. $\mathscr{C}_{\infty}(G)$ ), invariant under the translations. If $T$ is a weak*-(resp. norm-) closed homomorphism of $\mathscr{D}$ into $L^{\infty}(G)$ (resp. $\mathscr{C}_{\infty}(G)$ ) commuting with the translations, then there exists a $s_{0} \in G$ and a continuous one-parameter subgroup $\{g(t) ; t \in \mathbb{R}\}$ of $G$ such that for every $s \in G$ and $f \in \mathscr{D}$ there is an $F \in H^{\infty}(D)$ with boundary values:

$$
\left.\begin{array}{rl}
F(t) & =f(s+g(t)) \\
F(t-i) & =T f\left(s+s_{0}+g(t)\right), \quad t \in \mathbb{R} .
\end{array}\right\}
$$

(ii) In the above assertion, one may replace $L^{\infty}(G)$ and $\mathscr{C}_{\infty}(G)$ by $B(G)$ and $A(G)$ respectively, where the weak*-topology in $B(G)$ refers to the identification $B(G)=M(\hat{G})=\mathscr{C}_{\infty}(\hat{G})^{*}$.

\section{Density of the Two-Point Functions of Invariant States}

We begin by giving definitions and fixing notation. 
Definition 3.1. Given a $C^{*}$-algebra $\mathfrak{A}$ and a locally compact group $G$, an action of $G$ on $\mathfrak{A}$ is a homomorphism $g \rightarrow \alpha_{g}$ of $G$ into the automorphism group of $\mathfrak{A}$ such that the map $g \in G \rightarrow \alpha_{g}(A)$ is continuous for all $A \in \mathfrak{U}$. With an action $\alpha$ of $G$ on $\mathfrak{U}$ the triple $\{\mathfrak{U}, G, \alpha\}$ is called a $C^{*}$-system.

Definition 3.2. An $\alpha$-invariant state of the $C^{*}$-system $\{\mathfrak{U}, G, \alpha\}$ is a state $\omega$ of $\mathfrak{A}$ such that $\omega \circ \alpha_{g}=\omega$ for all $g \in G$.

The two-point functions $F_{A B}, G_{A B}$, resp. truncated two-point functions $f_{A B}, g_{A B}$, are defined as

$$
\left.\begin{array}{l}
F_{A B}(g)=\omega\left(B \alpha_{g}(A)\right) \\
G_{A B}(g)=\omega\left(\alpha_{g}(A) B\right)
\end{array}, \quad A, B \in \mathfrak{U},\right\}
$$

resp.

$$
\left.\begin{array}{l}
f_{A B}=F_{A B}-\omega(A) \omega(B) \mathbb{1} \\
g_{A B}=G_{A B}-\omega(A) \omega(B) \mathbb{1}
\end{array} \quad A, B \in \mathfrak{U} ;\right\}
$$

and we set

$$
\left.\begin{array}{rl}
\mathscr{F} & \left.=\left\{F_{A B} ; A, B \in \mathfrak{U}\right\}\right\} \\
\mathscr{G} & \left.=\left\{G_{A B} ; A, B \in \mathfrak{U}\right\}\right\} \\
\mathfrak{f} & =\left\{f_{A B} ; A, B \in \mathfrak{U}\right\} \\
\mathfrak{g} & \left.=g_{A B} ; A, B \in \mathfrak{U}\right\}
\end{array}\right\}
$$

In terms of the Gelfand-Neumark-Segal (GNS-)construktion $\left\{\pi, U, \mathscr{H}, \xi_{\omega}\right\}$ of $\omega$, the functions (3.1), (3.1a) respectively read

$$
\left.\begin{array}{l}
F_{A B}(g)=\left(\pi\left(B^{*}\right) \xi_{\omega}, U(g) \pi(A) \xi_{\omega}\right) \\
G_{A B}(g)=\left(\pi\left(A^{*}\right) \xi_{\omega}, U(g) \pi(B) \xi_{\omega}\right)
\end{array} \quad A, B \in \mathfrak{A}, g \in G,\right\}
$$

and

$$
\left.\begin{array}{l}
f_{A B}(g)=\left(\pi\left(B^{*}\right) \xi_{\omega}, U_{0}(g) \pi(A) \xi_{\omega}\right) \\
g_{A B}(g)=\left(\pi\left(A^{*}\right) \xi_{\omega}, U_{0}(g) \pi(B) \xi_{\omega}\right)
\end{array} \quad A, B \in \mathfrak{A}, g \in G .\right\}
$$

with $U_{0}$ the representation induced by $U$ in the subspace of $\mathscr{H}$ orthogonal to $\xi_{\omega}$. We see that the sets $\mathscr{F}, \mathscr{G}, \mathfrak{f}$, and $\mathfrak{g}$ are within the linear span $B(G)$ of $B^{+}(G)$, the set of continuous positive type functions on $G$. Setting ${ }^{6}$

$$
\begin{aligned}
B_{1}^{+}(G) & =\left\{\varphi \in B^{+}(G) ;\|\varphi\|_{\infty}=1\right\}, \\
\mathscr{F}^{+} & =\left\{F_{A A^{*}} ; A \in \mathfrak{U}\right\}, \quad \mathscr{F}_{1}^{+}=\left\{F_{A A^{*}} ; A \in \mathfrak{U}, \omega\left(A^{*} A\right)=1\right\}, \\
\tilde{\mathfrak{f}}^{+} & =\left\{f_{A A^{*}} ; A \in \mathfrak{U}\right\}, \quad \mathfrak{f}_{1}^{+}=\left\{f_{A A^{*}} ; A \in \mathfrak{U}, \omega\left(A^{*} A\right)-|\omega(A)|^{2}=1\right\},
\end{aligned}
$$

we note that $\mathscr{F}^{+}, \mathfrak{f}^{+} \subset B^{+}(G), \mathscr{F}_{1}^{+}, \mathfrak{f}_{1}^{+} \subset B_{1}^{+}(G)$.

We shall mainly be interested in the case where $G$ is abelian. $B(G)$ is then isomorphic to $M(\hat{G})=\mathscr{C}_{\infty}(\hat{G})^{*}$ and equipped as such with the weak* topology $\sigma\left(B(G), \mathscr{C}_{\infty}(\hat{G})\right)$. On the other hand $B(G)$ is immersed in $L^{\infty}(G)$, which we equip with its weak* topology $\sigma\left(L^{\infty}(G), L^{1}(G)\right)$. The two following facts are important for the

6 We recall that for $f \in B^{+}(G),\|f\|_{\infty}$ is the value of $f$ at the unit of $G$ 
sequel: $\sigma\left(L^{\infty}(G), L^{1}(G)\right)$ coïncides with the weak* topology of $B(G)$ on bounded ${ }^{7}$ subsets of the latter; and these topologies coïncide with the topology of uniform convergence on compacts on subsets of $B^{+}(G)$ bounded $^{7}$ above and below by positive constants (cf. [6], 2.7.5 and 13.5.2).

We finally note the properties (stated for $G$ abelian)

$$
\left.\begin{array}{l}
G_{A B}(g)=\bar{F}_{A^{*} B^{*}}(g) \\
G_{A B}(g)=F_{B A}(-g), \quad A, B \in \mathfrak{U}, g, s \in G, \\
F_{A B}(g+s)=F_{A \alpha_{s}(B)}(g) \\
G_{A B}(g+s)=G_{A \alpha_{s}(B)}(g)
\end{array}\right\}
$$

holding also if we replace $F_{A B}, G_{A B}$ by $f_{A B}$, resp. $g_{A B}$ : we see that $\mathscr{F}, \mathscr{G}, \tilde{f}$, and $\mathfrak{g}$ are (globally) translation invariant sets of functions.

Definition 3.3. Let, with the above notation, $\omega$ be an $\alpha$-invariant state of the $C^{*}$-system $\{\mathfrak{U}, G, \alpha\}$. Assume the group $G$ amenable non compact and denote by $\mathscr{M}(G)$ its set of invariant means [i.e. the set of states of the $C^{*}$-algebra $L^{\infty}(G)$ invariant under translations by elements of $G]$. Let, on the other hand, $\mathfrak{A}_{0}$ be a norm-dense, globally translation invariant *-subalgebra of $\mathfrak{A}$. We say that we have $\omega$-asymptotic abelianness whenever

$$
\left\|\pi\left\{\left(A \alpha_{g}(B)-\alpha_{g}(B) A\right) C\right\} \xi_{\omega}\right\| \underset{g=\infty}{\longrightarrow} 0, \quad A, B, C \in \mathfrak{U},
$$

and $L^{1}$-asymptotic abelianness on $\mathfrak{H}_{0}$ whenever

$$
\left\{g \rightarrow\left\|A \alpha_{g}(B)-\alpha_{g}(B) A\right\|\right\} \in L^{1}(G), \quad A, B \in \mathfrak{A}_{0} .
$$

On the other hand we say that the state $\omega$ is weakly $\alpha$-clustering whenever ${ }^{8}$

$$
\eta\left(f_{A B}\right)=0, \quad A, B \in \mathfrak{U}, \eta \in \mathscr{M}(G) ;
$$

$\alpha$-clustering of order $n$ ( $n$ a positive integer) whenever, to each set $A_{1}, \ldots, A_{p} \in \mathfrak{U}$, $p \leqq n$, there is a positive function $\varphi \in \mathscr{C}_{\infty}(G)$ with

$$
\left|\omega_{(p)}^{T}\left(\alpha_{g_{1}}\left(A_{1}\right), \ldots, \alpha_{g_{p}}\left(A_{p}\right)\right)\right| \leqq \varphi\left(g_{i}-g_{j}\right), \quad i \neq j
$$

(here $\omega_{(p)}^{T}$ denotes the truncated function of order $p$ see, e.g. [7]. VI, Appendix).

$L^{1}-\alpha$-clustering of order $n$ on $\mathfrak{U}_{0}$ whenever, to each set $A_{1}, \ldots, A_{p} \in \mathfrak{A}_{0}, p \leqq n$, there is a positive function $\varphi \in L^{1}(G)$ fulfilling (3.11).

We note that for $n=2$ the two last properties (to which we then simply refer as clustering, resp. $L^{2}$-clustering on $\mathfrak{U}_{0}$ ) reduce to

$$
f_{A B} \in \mathscr{C}_{\infty}(G), \quad A, B \in \mathfrak{U},
$$

resp.

$$
f_{A B} \in L^{1}(G), \quad A, B \in \mathfrak{U}_{0} .
$$

7 For the sup norm

8 It is well known that under the above asymptotic abelianness properties weak $\alpha$-clustering of $\omega$ is equivalent to extremal $\alpha$-invariance 
We now state our first density result:

Proposition 3.4. Let $\omega$, with the above notation, be an $\alpha$-invariant state of the $C^{*}$ system $\{\mathfrak{A}, G, \alpha\}$, where $G$ is abelian. We have that

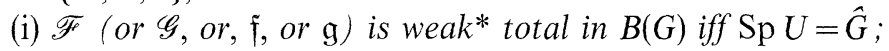

(ii) these sets are even weak* dense in $B(G)$ if we assume in addition the existence of an action $\tau$ of a locally compact group $H$ on $\mathfrak{U}$ commuting with $\alpha^{9}$ and such that $\omega$ is $\tau$-invariant and $\tau$-clustering (in particular if, in addition to $\operatorname{Sp} U=\hat{G}, \omega$ is $\alpha$ clustering).

(iii) assume $\omega \alpha$-clustering (so that $\mathfrak{f}, \mathfrak{g} \subset \mathscr{C}_{\infty}(G)$ ): then $\operatorname{Sp} U=\hat{G}$ iff $\mathfrak{f}$ (or $\mathfrak{g}$ ) is total in $\mathscr{C}_{\infty}(G)$ for the sup norm.

Proof. Owing to (3.6) we need only consider the case of $\mathscr{F}$ and $\mathfrak{f}$. The proof of (i) rests on the following known fact (see, e.g., [6], Sect. 18):

Let $V$, with spectrum $\Sigma \in \hat{G}$, be a continuous unitary representation of the abelian locally compact group $G$ on the Hilbert space $\mathscr{H}$ and let

$$
\mathscr{S}=\{\{g \in G \rightarrow(\varphi, V(g) \psi)\} ; \psi \in \mathfrak{f},\|\psi\|=1\},
$$

where $\mathfrak{F}$ is a dense linear subspace of $\mathscr{H}$. Then the weak* closed convex hull of $\mathscr{S}$ in $B(G)$ consists of the functions in $B_{1}^{+}(G)$ whose Fourier transforms have support within $\Sigma$.

Applying this to $V=U$ and $\mathfrak{f}=\pi(\mathfrak{U}) \xi_{\omega}$ [resp. $V=U$, and $\mathfrak{f}=E_{0} \pi(A) \xi_{\omega}, E_{0}$ the projection in $\mathscr{H}$ onto the subspace orthogonal to $\left.\xi_{\omega}\right]$, we see that the closed convex hull in $B(G)$ of the set $\mathscr{F}_{1}^{+}$, (resp. $\left.\mathfrak{f}_{1}^{+}\right)$is the whole $B_{1}^{+}(G)$. Assertion (i) then follows from the fact that $B_{1}^{+}(G)$, resp. $B^{+}(G)$, generate $B^{+}(G)$, resp. $B(G)$ by homogeneity, resp. linearity.

The proof of (ii) (as well as other arguments to come) follows a pattern described by

Remark 3.5. Let $X$ and $Y$ be topological spaces, with $(b, a) \in X \times Y \rightarrow b \circ a \in X$ a separately continuous map. If the respective subsets $S$ and $T$ of $X$ and $Y$ have closures $\bar{S}$ and $\bar{T}$ such that $T \circ S \subset \bar{S}$, one has $\bar{T} \circ \bar{S} \subset \bar{S}$ (immediate: let $x \in \bar{S}$ and $y \in \bar{T}$ be the respective limits of generalized sequences $\left\{x_{\sigma}\right\} \subset S$ and $\left\{y_{\tau}\right\} \subset T$ : one has successively $y_{\tau} \circ x_{\sigma} \underset{\sigma}{\longrightarrow} y_{\tau} \circ x \in \bar{S}$ for each fixed $\tau$; and $y_{\tau} \circ x \rightarrow y_{\tau} \circ x \in \bar{S}$ ).

Choosing $X=Y=B(G), S=T=\mathscr{F}$ or $\mathfrak{f}$, and $a \cdot b=a+b$, we see that (ii) follows if we prove that $\mathscr{F}+\mathscr{F} C \mathscr{\mathscr { F }}$ and $\mathfrak{f}+\mathfrak{f} \subset \overline{\mathfrak{f}}$.

Now $\tau$-clustering of $\omega$ entails that one has, for all $A_{1}, A_{2}, B_{1}, B_{2} \in \mathfrak{A}$,

$$
\begin{aligned}
& F_{A_{1}+\tau_{u}\left(A_{2}\right), B_{1}+\tau_{2 u}\left(B_{2}\right)} \underset{u=\infty}{\longrightarrow} F_{A_{1} B_{1}}+\left[\omega\left(A_{1}\right) \omega\left(B_{2}\right)+\omega\left(A_{2}\right) \omega\left(B_{1}+B_{2}\right)\right] \mathbb{1}, \\
& F_{A_{1}+\tau_{u}\left(A_{2}\right), B_{1}+\tau_{u}\left(B_{2}\right)} \underset{u=\infty}{\longrightarrow} F_{A_{1} B_{1}}+F_{A_{2} B_{2}}+\left[\omega\left(A_{1}\right) \omega\left(B_{2}\right)+\omega\left(B_{1}\right) \omega\left(A_{2}\right)\right] \mathbb{1}, \\
& f_{A_{1}+\tau_{u}\left(A_{2}\right), B_{1}+\tau_{u}\left(B_{2}\right)} \underset{u=\infty}{\longrightarrow} f_{A_{1} B_{1}}+\mathrm{f}_{A_{2} B_{2}},
\end{aligned}
$$

poinwise and, since the functions on the left are uniformly bounded, in the $\sigma\left(L^{\infty}(G)\right.$, $\left.L^{1}(G)\right)$ and $\sigma\left(B(G), \mathscr{C}_{\infty}(\hat{G})\right)$ topologies. Now (3.17) shows that $\mathfrak{f}+\mathfrak{f} \subset \mathfrak{F}$. Further we see

$9 \quad$ I.e. $\tau_{h} \circ \alpha_{g}=\alpha_{g} \circ \tau_{h}, g \in \mathrm{G}, \mathrm{h} \in \mathrm{H}$ 
from (3.15) $F_{A_{1} B_{1}}+\lambda \mathbb{1} \in \overline{\mathscr{F}}$ for all $A_{1}, B_{1} \in \mathfrak{A}$ and $\lambda \in \mathbb{C}$ [choose $A_{2}, B_{2}$ with $\omega\left(\mathrm{B}_{1}\right.$ $\left.+\mathrm{B}_{2}\right) \neq 0$ and $\left.\omega\left(A_{2}\right)=\left[\lambda-\omega\left(A_{1}\right) \omega\left(B_{2}\right)\right] / \omega\left(B_{1}+B_{2}\right)\right]$. This combined with (3.16) shows that $\mathscr{F}+\mathscr{F}(\overline{\mathscr{F}}$.

We now prove (iii): $\operatorname{Sp} U=\hat{G}$ entails $\operatorname{Sp} U_{0}=\hat{G}$, from which follows that $U_{0}(f)$ $=0, f \in L^{1}(G)$, iff $f=0\left[U_{0}\right.$ denotes also the associated representation of $L^{1}(G)$ and $M(G)]$. Let now $\mu \in M(G)$ be non vanishing: there is an $f \in L^{1}(G)$ with $\mu * f \neq 0$ and thus $U_{0}(\mu * f)=U_{0}(\mu) U_{0}(f) \neq 0$, whence $U_{0}(\mu) \neq 0$. But, from (3.3a) we have

$$
\left(\pi\left(B^{*}\right) \xi_{\omega}, U_{0}(\mu) \pi(A) \xi_{\omega}\right)=\int f_{A B}(g) d \mu(g)=\left\langle\mu, f_{A B}\right\rangle, \quad A, B \in \mathfrak{A},
$$

and $\xi_{\omega}$ is cyclic: thus $\mu$ vanishes on $\mathfrak{f}$ iff $U_{0}(\mu)=0$ : we see that $\mathfrak{f}$ is total in $\mathscr{C}_{\infty}(G)$ iff $\operatorname{Sp} I=\hat{G}$.

Remark 3.6. We also proved that in the frame of Proposition 3.4 one has $\operatorname{Sp} U=\hat{G}$ iff the weak* closed convex hull of any of the sets $\mathscr{F}^{+}, \mathscr{G}^{+}, \mathfrak{f}^{+}, \mathfrak{g}^{+}\left(\mathscr{\mathscr { F }}_{1}^{1+}, \mathscr{G}_{1}^{+}, \mathfrak{f}_{1}^{+}\right.$, $\left.\mathfrak{g}_{1}^{+}\right)$coïncides with $B^{+}(G)\left(B_{1}^{+}(G)\right)$; and further that if $\omega$ is $\tau$-clustering, the weak* closures of $\mathscr{F}^{+}, \mathscr{G}^{+}, \mathfrak{f}^{+}$, and $\mathfrak{g}^{+}$are already convex.

Our next result pertains to density in $L^{\infty}(G)$.

Proposition 3.7. Let $\omega$, with the above notation, be an $\alpha$-invariant state of the $C^{*}$ system $\{\mathfrak{U}, G, \alpha\}$. And assume the existence of an action $\tau$ of an amenable group $H$ on $\mathfrak{A}$, commuting with $\alpha, \omega$-asymptotically abelian, and for which $\omega$ is weakly $\tau$ clustering. If $\mathrm{Sp} U=\hat{G}$ the sets $\mathscr{\mathscr { F }}, \mathscr{G}, \mathfrak{f}, g$ are all weak ${ }^{*}$ total in $L^{\infty}(G)$. These sets are even dense in $L^{\infty}(G)$ if $\omega$ is $\tau$-clustering.

Proof. We need only consider $\mathscr{F}$ and $\mathfrak{\uparrow}$ whose weak* closed linear hulls in $L^{\infty}(G)$ we denote $[\mathscr{F}]^{\infty}$, resp. $[\mathfrak{f}]^{\infty}$. As a first step we shall prove that the latter are multiplicative. For this we observe that by Remark 3.6 with $X=Y=L^{\infty}(G), S=T$ $=\mathscr{F}$ or $\mathfrak{f}$, and $a \cdot b=a b$, it suffices to show that $\mathscr{F} \cdot \mathscr{F} \subset[\mathscr{F}]^{\infty}$ and $\mathfrak{f} \cdot \mathfrak{f} \subset[\mathfrak{f}]^{\infty}$. This will result from the assumed clustering and asymptotic abelianness.

Consider the functions

$$
\begin{aligned}
& \left.\Phi_{u}=F_{A_{1} \tau_{u}\left(A_{2}\right), B_{1} \tau_{u}\left(B_{2}\right)}, \quad A_{1}, A_{2}, B_{1}, B_{2} \in \mathfrak{A}, u \in H\right\} . \\
& \varphi_{u}=f_{A_{1} \tau_{u}\left(A_{2}\right), B_{1} \tau_{u}\left(B_{2}\right)}
\end{aligned}
$$

and let $\eta \in \mathscr{M}(H)$. Since the representation of $L^{\infty}(H)$ by pointwise multiplication on $L^{2}(H)$ is faithful ${ }^{10}$ there is a net $\left\{\eta_{\sigma}\right\} \subset L^{1}(H)$ such that $\eta_{\sigma} \rightarrow \eta$ in the weak* topology of $L^{\infty}(H)^{*}$. Smearing out the functions (3.19) with the $\eta_{\sigma}$ :

$$
\left.\begin{array}{l}
\Phi_{\sigma}=\int_{H} \Phi_{u} \eta_{\sigma}(\mathrm{u}) \mathrm{du} \\
\varphi_{\tau}=\int_{H} \varphi_{u} \eta_{\sigma}(\mathrm{u}) \mathrm{du}
\end{array}\right\}
$$

we obtain, as shown by the Fubini theorem, respective elements of $\mathscr{F} \perp \perp=[\mathscr{F}]^{\infty}$ and $\mathfrak{f}^{\perp \perp}=[\mathfrak{f}]^{\infty}$ yielding the pointwise limits

$$
\left.\begin{array}{l}
\Phi_{\sigma} \underset{\sigma}{\rightarrow} F_{A_{1} B_{1}} \cdot F_{A_{2} B_{2}} \\
\varphi_{\sigma} \underset{\sigma}{\rightarrow} f_{A_{1} B_{1}} \cdot f_{A_{2} B_{2}}+\omega\left(A_{2}\right) f_{A_{1} B_{1}}+\omega\left(A_{1}\right) f_{A_{2} B_{2}}
\end{array}\right\}
$$

10 See [6] 
as follows from the weak $\tau$-clustering of $\omega$, owing to

$$
\begin{aligned}
\Phi_{u}(g) & =\varphi_{u}(g)+\omega\left(A_{1} \tau_{u}\left(A_{2}\right) \omega\left(B_{1} \tau_{u}\left(B_{2}\right)\right)\right. \\
& =\omega\left(B_{1} \alpha_{g}\left(A_{1}\right) \tau_{u}\left(B_{2} \alpha_{g}\left(A_{2}\right)\right)\right)+\omega\left(B_{1}\left(\tau_{u}\left(B_{2}\right) \alpha_{g}\left(A_{1}\right)-\alpha_{g}\left(A_{1}\right) \tau_{u}\left(B_{2}\right)\right) \tau_{u} \alpha_{g}\left(A_{2}\right)\right)
\end{aligned}
$$

since $\eta$ vanishes on the last term, due to $\omega$-asymptotic abelianness for the action $\tau$ : (3.21) now entails $\mathscr{F} \cdot \mathscr{F} \subset[\mathscr{F}]^{\infty}, \mathfrak{f} \cdot \mathfrak{f} \subset[\mathfrak{f}]^{\infty}$, observing that $\Phi_{\sigma}$ and $\varphi_{\sigma}$ are bounded by $\left\|A_{1}\right\| \cdot\left\|A_{2}\right\| \cdot\left\|B_{1}\right\| \cdot\left\|B_{2}\right\|$, and thus that the limits hold in $\sigma\left(L^{\infty}(G), L^{1}(G)\right)$ by dominated convergence.

From the multiplicativity of $[\mathscr{F}]^{\infty}$ and $[\mathrm{f}]^{\infty}$, and from (3.6), (3.7) we now conclude that $[\mathscr{F}]^{\infty} \cap[\mathscr{G}]^{\infty}$ and $[\mathrm{f}]^{\infty} \cap[\mathfrak{g}]^{\infty}$ are both globally translation invariant von Neumann subalgebras of $L^{\infty}(G)$. Theorem 2 of [8] then asserts that they are describable as subsets of $L^{\infty}$-functions invariant under some closed subgroup of $G$ : but the latter has to be trivial due to $\operatorname{Sp} U=\operatorname{Sp} U_{0}=\hat{G}$. Thus $[\mathscr{F}]^{\infty}$ $=[\mathscr{G}]^{\infty}=[\mathfrak{f}]^{\infty}=[\mathfrak{g}]^{\infty}=L^{\infty}(G)$. And we proved earlier [cf. proof of Proposition 3.4 (ii)] that if $\omega$ is $\tau$-clustering the weak* closures of these sets are already linear.

We now prove density in $L^{1}(G)$ under a sharper clustering assumption (for a neighbouring result, see [9]).

Proposition 3.8. Let $\omega$ be an $\alpha$-invariant state of the $C^{*}$-system $\{\mathfrak{U}, G, \alpha\}$, with $G$ abelian and $\mathfrak{P}$ unital. If $\omega$ is $L^{1}-\alpha$-clustering of order 4 , and $\operatorname{Sp} U=\hat{G} \mathfrak{f}$ and $\mathfrak{g}$ are both total in $L^{1}(G)$.

Proof. Since $\omega$ is a fortiori $L^{1}$-clustering, we have $\mathfrak{f}, \mathfrak{g} \subset L^{1}(G)$. Let [f] $]_{1}$ be the closed convex hull of $\mathfrak{f}$ for the $L^{1}$-norm, we must show that $[\mathfrak{f}]_{1}=L^{1}(G)$. Since $\mathfrak{f}$ is globally translation invariant, $[\mathfrak{f}]_{1}$ is a closed ideal of the group algebra $L^{1}(G)$. Thus if $\Sigma_{0}$ is the set of points of $\hat{G}$ at which the Fourier transform of at least one element of $[\mathfrak{f}]_{1}$ does not vanish, we shall have $[\mathfrak{f}]_{1}=L^{1}(G)$ if we show that $\Sigma_{0}=\hat{G}$. Now this will hold if we show that $[\mathfrak{f}]_{1}$ is stable under multiplications by all elements of $L^{\infty}(G)$, since, taking for the latter all characters of $G, \Sigma_{0}$ is seen to be translation invariant in $\hat{G}$, and hence to cover $\hat{G}$ due to $\operatorname{Sp} U_{0}=\hat{G}$.

Our proof thus boils down to proving that $L^{\infty}(G) \cdot[\mathfrak{f}]_{1} \subset[\mathfrak{f}]_{1}$, i.e. $[\mathfrak{f}]^{\infty}$ $\cdot[\mathfrak{f}]_{1} \subset[\mathfrak{T}]_{1}$ by Proposition 3.7. Now Remark 3.5 reduces this to showing that $\tilde{f} \cdot \tilde{f} \subset[\mathfrak{T}]_{1} \quad\left[\right.$ choose $X=L^{1}(G)$ with its weak topology, $Y=L^{\infty}(G)$ with its weak topology, $S=T=\tilde{f}, a \circ b=a b$; and observe that weakly closed and norm closed linear hulls in $L^{1}(G)$ coïncide].

The fact that $\mathfrak{f} \cdot \mathfrak{f} \subset[\mathfrak{f}]_{1}$ will now result from the $L^{1}-\alpha$-clustering of $\omega$ by a sharpening of the argument establishing Proposition 3.7. We shall use the function $\varphi_{u}$ in (3.19) with $\tau=\alpha$ and a modified form of $A_{1}, A_{2}, B_{1}, B_{2}$. Note that if we define

$$
A^{\prime}=A-\omega(A) \mathbb{1}, \quad A \in \mathfrak{U},
$$

we have the following straightforward properties

$$
\begin{aligned}
& \omega\left(A^{\prime}\right)=0, \quad A \in \mathfrak{U}, \\
& \alpha_{g}\left(A^{\prime}\right)=\alpha_{g}(A)^{\prime}, \quad A \in \mathfrak{U}, g \in G, \\
& \omega_{(p)}^{T}\left(A_{1}^{\prime}, \ldots, A_{p}^{\prime}\right)=\omega_{(p)}^{T}\left(A_{1}, \ldots, A_{p}\right), \quad A_{i} \in \mathfrak{U}, \\
& f_{A^{\prime} B^{\prime}}(g)=\omega_{(2)}^{T}\left(B, \alpha_{g}(A)\right)=f_{A B}(g), \quad A, B \in \mathfrak{U}, g \in G,
\end{aligned}
$$


$[(3.26)$ is immediate from the fact that any truncated function vanishes whenever one of its arguments is a multiple of the unit]. From (3.26) and (3.25) follows that if (3.11) holds, it also holds with (some of) the $A_{i}$ replaced by the corresponding $A_{i}^{\prime}$ Let now $A_{1}, A_{2}, B_{1}, B_{2} \in \mathfrak{U}$ and expand in terms of truncated functions the first term in the r.h.s. of the second line in 11

$$
\begin{aligned}
\varphi_{u}^{\prime}(g) & =f_{A_{1} \alpha_{u}\left(A_{2}\right), B_{1} \alpha_{u}\left(B_{2}\right)} \\
& =\omega\left(B_{1}^{\prime} \alpha_{u}\left(B_{2}^{\prime}\right) \alpha_{g}\left(A_{1}^{\prime}\right) \alpha_{g+u}\left(A_{2}^{\prime}\right)\right)-\omega\left(A_{1}^{\prime} \alpha_{u}\left(A_{2}^{\prime}\right)\right) \omega\left(B_{1}^{\prime} \alpha_{u}\left(B_{2}^{\prime}\right)\right) .
\end{aligned}
$$

We obtain taking account of (3.24) through (3.27) above

$$
\begin{aligned}
\varphi_{u}^{\prime}(g)= & f_{A_{1} B_{1}}(g) f_{A_{2} B_{2}}(g)+\omega_{(4)}^{T}\left(B_{1} \alpha_{u}\left(B_{2}\right) \alpha_{g}\left(A_{1}\right) \alpha_{g+u}\left(A_{2}\right)\right) \\
& +\omega_{(2)}^{T}\left(B_{1} \alpha_{u+g}\left(A_{2}\right)\right) \omega_{(2)}^{T}\left(\alpha_{u}\left(B_{2}\right) \alpha_{g}\left(A_{1}\right)\right),
\end{aligned}
$$

where, due to the assumed $L^{1}-\alpha$-clustering of $\omega$ of order 4 , the two last terms are majorized by a fixed $L^{1}$-function of $g$, and tend to zero for $u \rightarrow \infty$. The dominated convergence theorem then implies that $\varphi_{u}^{\prime} \underset{u=\infty}{\longrightarrow} \mathrm{f}_{A_{1} B_{1}} \cdot \mathrm{f}_{A_{2} B_{2}}$, whence $\mathfrak{f} \cdot \tilde{f} \subset[\mathfrak{f}]_{0}$.

We end up this section by collecting results in the physically important case $G=\mathbb{R}$, the additive group of reals, where asymptotic abelianness and weak clustering are known [10] to imply a "spectral alternative".

Proposition 3.9. Let $\omega$ be an extremal invariant state of a $C^{*}$-system $\{\mathfrak{H}, \mathbb{R}, \alpha\}$ assumed $\omega$-asymptotically abelian. Adopt the notation of Definition 3.2 and denote by []$_{*},[]^{\infty},[]_{1},[]_{\infty}$ closed linear hulls in respectively in $B(\mathbb{R}), L^{\infty}(\mathbb{R})$ for their weak* topologies and $L^{1}(\mathbb{R}), \mathscr{C}_{\infty}(\mathbb{R})$ for their norm topologies.

One has then the following alternative (i), (ii), (iii):

(i) $\mathrm{Sp} U=\mathbb{R} ;[\mathscr{F}]_{*}=[\mathscr{G}]_{*}=[\mathfrak{f}]_{*}=[\mathfrak{g}]_{*}=B(\mathbb{R})$; and $[\mathscr{F}]^{\infty}=[\mathscr{G}]^{\infty}=[\mathfrak{f}]^{\infty}$ $=[\mathfrak{g}]^{\infty}=L^{\infty}(\mathbb{R})$;

(ii) $\mathrm{Sp} U=\{n a ; n \in \mathbb{Z}\}$ for some $a>0 ; \pi(\mathfrak{U})$ is abelian; $[\mathscr{F}]_{*}=[\mathscr{G}]_{*}=[\mathfrak{f}]_{*}$ $=[\mathfrak{g}]_{*}=\{f \in B(\mathbb{R}) ; f$ is periodic with period $2 \pi / a\}$

$[\mathscr{F}]^{\infty}=[\mathscr{G}]^{\infty}=[\mathfrak{f}]^{\infty}=[\mathfrak{g}]^{\infty}=\left\{f \in L^{\infty}(G) ; f\right.$ is periodic with period $\left.2 \pi / a\right\}$;

(iii) $\operatorname{Sp} U \cap(-\operatorname{Sp} U)=\emptyset$ and $[\mathscr{F}]_{*} \cap[\mathscr{G}]_{*},[\mathfrak{f}]_{*} \cap[\mathfrak{g}]_{*},[\mathscr{F}]^{\infty} \cap[\mathscr{G}]^{\infty}$, and $[\mathfrak{f}]^{\infty} \cap[\dot{\mathfrak{g}}]^{\infty}$ all reduce to the constant functions.

Moreover, if we assume that $\omega$ is $\alpha$-clustering, the weak*-closures of $\mathscr{F}, \mathscr{G}, \mathfrak{f}$ and $\mathfrak{g}$ in both $B(\mathbb{R})$ and $L^{\infty}(\mathbb{R})$ are already linear (thus coincide with the above closed convex hulls); alternative (ii) is excluded; and $[\mathfrak{f}]_{\infty}=[\mathfrak{g}]_{\infty}=\mathscr{C}_{\infty}(\mathbb{R})$.

Finally, if $\operatorname{Sp} U=\mathbb{R}$ and if $\omega$ is $L^{1}$-clustering of order $4,[\mathfrak{f}]_{1}=[\mathfrak{g}]_{1}=L^{1}(\mathbb{R})$.

The density proofs for case (ii) are analogous to those presented earlier for case (i); and the result in case (iii) follows from the fact that the set of Hardy functions intersects its complex conjugate along the constant functions.

\section{Criteria for the KMS Property}

Combining the duality results of Sect. 2 (specifically Corollary 2.6) with the density results of Sect. 3, we now obtain criteria for the KMS nature of invariant states of

11 For the full expression of this expansion in the general case, see the Appendix 
$C^{*}$-systems with varying degrees of clustering. For weakly clustering states we have

Theorem 4.1. Let $\omega$, with the notation of Definition 3.2, be an $\alpha$-invariant state of the $C^{*}$-system $\{\mathfrak{U}, G, \alpha\}$, with $G$ abelian and $\operatorname{Sp} U=\hat{G}$. Assume that $\omega$ is extremal $\tau$ invariant for an action $\tau$ of an amenable group $H$ on $\mathfrak{A}$ which is $\omega$-asymptotically abelian and commutes with $\alpha$. Denote by $\mathscr{A}$ either $L^{\infty}(G)$ topologized by $\sigma\left(L^{\infty}(G)\right.$, $L^{1}(G)$ ) or $B(G)$ topologized by $\sigma\left(B(G), \mathscr{C}_{\infty}(\hat{G})\right.$ ). If there is a closable linear operator $T_{0}$ in $\mathscr{A}$, with domain the linear span of $\mathscr{F}(\tilde{\mathfrak{T}})^{12}$, such that $T_{0} F_{A B}=G_{A B}\left(T_{0} f_{A B}=g_{A B}\right)$ for all $A, B \in \mathfrak{U}$, then $\omega$ is KMS for some continuous one-parameter subgroup of $G^{13}$.

Proof ${ }^{14}$. The conclusion will follow if we show that the closure $T$ of $T_{0}$ can be taken as the operator $T$ in Corollary 2.6: indeed, from (3.5) and (3.6) we have that $T$ commutes with $f \rightarrow f^{*}$, where $f^{*}(s)=\bar{f}(-s), s \in G$, entailing $s_{0}=0$ in the conclusion of Corollary 2.7.

Let $\mathscr{D}_{T}$ be the domain of $T$ : since it includes the linear span of $\mathscr{F}(\mathfrak{f})$, it is dense in $\mathscr{A}$ by Propositions 3.4 and 3.7. On the other hand, it is clear from (3.7) that $\mathscr{D}_{T}$ is stable under all translations by elements of $G$ and that $T$ commutes with these translations. Thus we are left with the proof that $\mathscr{D}_{T}$ is closed for products and that $T$ is multiplicative. For this (and later arguments) we need the following general fact:

Remark 4.2. Let $\mathscr{A}$ be a topological space with $\left(f_{1}, f_{2}\right) \in \mathscr{A} \times \mathscr{A} \rightarrow f_{1} \circ f_{2} \in \mathscr{A}$ a separately continuous map. Let $T_{0}: \mathscr{S} \subset \mathscr{A} \rightarrow \mathscr{A}$ be a closable operator and assume that the closure $T$ of $T_{0}$ fulfills $\mathscr{S} \circ \mathscr{S} \subset \mathscr{D}_{T}$ and $T\left(f_{1} \circ f_{2}\right)=\left(T_{0} f_{1}\right) \circ\left(T_{0} f_{2}\right), f_{1}, f_{2} \in \mathscr{S}$. One then has for all $\varphi_{1}, \varphi_{2} \in \mathscr{D}_{T}, \varphi_{1} \circ \varphi_{2} \in \mathscr{D}_{T}$ with $T\left(\varphi_{1} \circ \varphi_{2}\right)=\left(T \varphi_{1}\right) \circ\left(T \varphi_{2}\right)$.

The proof of this fact is immediate: let, for $\varphi_{1}, \varphi_{2} \in \mathscr{D}_{T},\left\{\varphi_{1}^{\sigma}\right\}$ and $\left\{\varphi_{2}^{\tau}\right\}$ be generalized sequences in $\mathscr{S}$ such that

$$
\left.\begin{array}{lll}
\varphi_{1}^{\sigma} \underset{\sigma}{\rightarrow} \varphi_{1} & \text { whilst } & T_{0} \varphi_{1}^{\sigma} \underset{\sigma}{\rightarrow} T \varphi_{1} \\
\varphi_{2}^{\tau} \underset{\tau}{\rightarrow} \varphi_{2} & \text { whilst } & T_{0} \varphi_{2}^{\sigma} \underset{\tau}{\longrightarrow} T \varphi_{2}
\end{array}\right\}
$$

By assumption $\varphi_{1}^{\tau} \circ \varphi_{2}^{\sigma} \in \mathscr{D}_{T}$ with $T\left(\varphi_{1}^{\sigma} \circ \varphi_{2}^{\tau}\right)=\left(T_{0} \varphi_{1}^{\tau}\right) \circ\left(T \varphi_{2}^{\tau}\right)$. Thus (4.1) implies successively, by separate continuity

$$
\varphi_{1}^{\sigma} \circ \varphi_{2}^{\tau} \underset{\sigma}{\rightarrow} \varphi_{1} \circ \varphi_{2}^{\tau} \quad \text { whilst } \quad T\left(\varphi_{1}^{\tau} \circ \varphi_{2}^{\tau}\right) \underset{\sigma}{\rightarrow}\left(T \varphi_{1}\right) \circ\left(T_{0} \varphi_{2}^{\tau}\right)
$$

for each fixed $\tau$, whence $\varphi_{1} \circ \varphi_{2}^{\tau} \in \mathscr{D}_{T}$ with $T\left(\varphi_{1} \circ \varphi_{2}^{\tau}\right)=\left(T \varphi_{1}\right) \circ\left(T_{0} \varphi_{2}^{\tau}\right)$, and

$$
\varphi_{1} \circ \varphi_{2}^{\tau} \underset{\tau}{\rightarrow} \varphi_{1} \circ \varphi_{2} \quad \text { whilst } T\left(\varphi_{1} \circ \varphi_{2}^{\tau}\right) \underset{\tau}{\longrightarrow}\left(T \varphi_{1}\right) \circ\left(T \varphi_{2}\right) \text {. }
$$

We now resume our proof, which by the preceding Remark (with $\mathscr{S}$ the linear $\operatorname{span}[\mathscr{F}]$ of $\mathscr{F}([\mathfrak{T}]$ of $\mathfrak{f})$ and $\left.f_{1} \circ f_{2}=f_{1} f_{2}\right)$ boils down to the proof of

$$
f_{1} f_{2} \in \mathscr{D}_{T} \text { with } T\left(f_{1} f_{0}\right)=\left(T_{0} f_{1}\right) \circ\left(T_{0} f_{2}\right), \quad f_{1}, f_{2} \in[\mathscr{F}]([\mathfrak{\dagger}]) .
$$

12 The theorem remains valid if one replaces $\mathscr{F}(\mathfrak{f})$ by the set $\mathscr{F}_{0}\left(\tilde{f}_{0}\right)$ obtained by restricting $A, B$ to a translation invariant, norm dense $*$-subalgebra $\mathfrak{U}_{0}$ of $\mathfrak{A}$

13 If $G=\mathbb{R}$, the additive group of reals, the conclusion means that $\omega$ is $\beta$-KMS for some real $\beta$

14 The proof is effected at the same time for the two choices $\mathscr{A}=L^{\infty}(G)$ and $\mathscr{A}=B(G)$ 
By linearity, this reduces to

$$
\begin{array}{lll}
F_{A_{1} B_{1}} \cdot F_{A_{2} B_{2}} \in \mathscr{D}_{T} & \text { with } & T\left(F_{A_{1} B_{1}} \cdot F_{A_{2} B_{2}}\right)=G_{A_{1} B_{1}} \cdot G_{A_{2} B_{2}} A_{1}, A_{2}, B_{1}, B_{2} \in \mathfrak{U} \\
f_{A_{1} B_{1}} \cdot f_{A_{2} B_{2}} \in \mathscr{D}_{T} & \text { with } & T\left(f_{A_{1} B_{1}} \cdot f_{A_{2} B_{2}}\right)=g_{A_{1} B_{1}} \cdot g_{A_{2} B_{2}},
\end{array}
$$

a property which will result from asymptotic abelianness and clustering. To show this, we consider again the functions (3.19), (3.20), together with their counterparts

$$
\left.\begin{array}{l}
\Psi_{u}=G_{A_{1} \tau_{u}\left(A_{2}\right), B_{1} \tau_{u}\left(B_{2}\right)} \\
\psi_{u}=g_{A_{1} \tau_{u}\left(A_{2}\right), B_{1} \tau_{u}\left(B_{2}\right)}, \quad A_{1}, A_{2}, B_{1}, B_{2} \in \mathfrak{U}, u \in H,
\end{array}\right\}
$$

and

$$
\left.\begin{array}{l}
\Psi_{\sigma}=\int_{H} \varphi_{u} \eta_{\sigma}(\mathrm{u}) \mathrm{du} \\
\psi_{\sigma}=\int_{H} \psi_{u} \eta_{\sigma}(\mathrm{u}) \mathrm{du}
\end{array}\right\}
$$

whereby the $\eta_{\sigma}$ are now chosen continuous with compact support [possible, since one can approximate weakly the state $\eta$ of $\left.L^{\infty}(H)\right]$ using the dense subset $\mathscr{C}_{K}(H)$ of $L^{2}(H)$. This allows us, approximating the integrals (3.20) and (4.7) by finite sums ${ }^{15}$, to write

$$
\left.\begin{array}{ll}
\Phi_{\sigma}=\lim _{n} \Phi_{n}, & \Psi_{\sigma}=\lim _{n} T_{0} \Phi_{n} \\
\varphi_{\sigma}=\lim _{n} \varphi_{n}, & \psi_{\sigma}=\lim _{n} T_{0} \varphi_{n},
\end{array}\right\}
$$

where the sequences at the right consist of uniformly bounded elements of $[\mathscr{F}]$ resp. [i]], and the limits can thus be taken in the weak* topology of $\mathscr{A}$ : from this follows that

$$
\left.\begin{array}{ll}
\Phi_{\sigma} \in \mathscr{D}_{T}, & T \Phi_{\sigma}=\Psi_{\sigma} \\
\varphi_{\sigma} \in \mathscr{D}_{T}, & T \varphi_{\sigma}=\psi_{\sigma} \cdot
\end{array}\right\}
$$

On the other hand $\omega$-asymptotic abelianness and weak $\tau$-clustering of $\omega$ entail that, for all $A_{1}, A_{2}, B_{1}, B_{2}$.

$$
\left.\begin{array}{l}
\Phi_{\sigma} \underset{\tau}{\rightarrow} F_{A_{1} B_{1}} \cdot F_{A_{2} B_{2}} \\
\Psi_{\sigma} \underset{\sigma}{\rightarrow} G_{A_{1} B_{1}} \cdot G_{A_{2} B_{2}}
\end{array}\right\}
$$

and

$$
\left.\begin{array}{l}
\varphi_{\sigma} \underset{\sigma}{\rightarrow} f_{A_{1} B_{1}} \cdot f_{A_{2} B_{2}}+\omega\left(A_{2}\right) \omega\left(B_{2}\right) f_{A_{1} B_{1}}+\omega\left(A_{1}\right) \omega\left(B_{1}\right) f_{A_{2} B_{2}} \\
\psi_{\sigma} \underset{\sigma}{\rightarrow} g_{A_{1} B_{1}} \cdot g_{A_{2} B_{2}}+\omega\left(A_{2}\right) \omega\left(B_{2}\right) g_{A_{1} B_{1}}+\omega\left(A_{1}\right) \omega\left(B_{1}\right) g_{A_{2} B_{2}},
\end{array}\right\}
$$

whence $(4.5),(4.5 \mathrm{a})$ since these limits again hold in the weak* topology of $\mathscr{A}$ because the nets at the left are uniformly bounded.

15 Observe that the Dirac measures are total in $M(H)$ 
Theorem 4.1 can be sharpened as follows if $\omega$ is assumed $\tau$-clustering:

Theorem 4.3. The conclusion of Theorem 4.1 is maintained under the following changes in the assumptions

(i) $\omega$ is assume $\tau$-invariant and $\tau$-clustering (instead of only extremal $\tau$ invariant);

(ii) $T_{0}$ is merely defined on $\mathscr{F}(\mathfrak{f})$ and closable in $\mathscr{A}$ (the linearity assumption for $T_{0}$, and its domain, is suppressed).

Proof. We reduce this result to Theorem 4.1 by showing that the $\tau$-clustering assumption automatically implies that $\mathscr{D}_{T}$ is linear and $T$ linear on it. Homogeneity is trivially shown. And the proof of linearity is reduced by Remark 4.2 (with $\mathscr{A}$ and $\mathscr{S}$ unchanged but $f_{1} \circ f_{2}=f_{1}+f_{2}$ ) to the proof of

$$
\left.\begin{array}{l}
F_{A_{1} B_{1}}+F_{A_{2} B_{2}} \in \mathscr{D}_{T} \text { with } \\
T\left(F_{A_{1} B_{1}}+F_{A_{2} B_{2}}\right)=G_{A_{1} B_{1}}+G_{A_{2} B_{2}},
\end{array} \quad A_{1}, A_{2}, B_{1}, B_{2} \in \mathfrak{U}\right\},
$$

resp. the same fact for the $f_{A B}$ instead of the $F_{A B}$. To establish this, we use the functions in (3.15)-(3.17) and their counterparts. The $\tau$-clustering of $\omega$ entails

$$
\left.\begin{array}{l}
F_{A_{1}+\tau_{u}\left(A_{2}\right), B_{1}+\tau_{2 u}\left(B_{2}\right)} \underset{u=\infty}{\longrightarrow} F_{A_{1} B_{1}}+\lambda \mathbb{I} \\
G_{A_{1}+\tau_{u}\left(A_{2}\right), B_{1}+\tau_{2 u}\left(B_{2}\right)} \underset{u=\infty}{\longrightarrow} G_{A_{1} B_{1}}+\lambda \mathbb{1},
\end{array} \quad A_{1}, B_{1} \in \mathfrak{U}\right\}
$$

with the choice of $A_{2}, B_{2}$ made in the proof of Proposition 3.4(ii) in order to match a given $\lambda \in \mathbb{C}$ on the r.h.s.; further

$$
\left.\begin{array}{l}
F_{A_{1}+\tau_{u}\left(A_{2}\right), B_{1}+\tau_{u}\left(B_{2}\right)}-\left[\omega\left(A_{1}\right) \omega\left(B_{2}\right)+\omega\left(B_{1}\right) \omega\left(A_{2}\right)\right] \mathbb{1} \underset{u=\infty}{\longrightarrow} F_{A_{1} B_{1}}+F_{A_{2} B_{2}} \\
G_{A_{1}+\tau_{u}\left(A_{2}\right), B_{1}+\tau_{u}\left(B_{2}\right)}-\left[\omega\left(A_{1}\right) \omega\left(B_{2}\right)+\omega\left(B_{1}\right) \omega\left(A_{2}\right)\right] \mathbb{1} \underset{u=\infty}{\longrightarrow} G_{A_{1} B_{1}}+G_{A_{2} B_{2}}
\end{array}\right\}
$$

and

$$
\left.\begin{array}{l}
f_{A_{1}+\tau_{u}\left(A_{2}\right), B_{1}+\tau_{u}\left(B_{2}\right)} \underset{u=\infty}{\longrightarrow} f_{A_{1} B_{1}}+f_{A_{2} B_{2}} \\
g_{A_{1}+\tau_{u}\left(A_{2}\right), B_{1}+\tau_{u}\left(B_{2}\right)} \underset{u=\infty}{\longrightarrow} g_{A_{1} B_{1}}+g_{A_{2} B_{2}}
\end{array}\right\}
$$

for all $A_{1}, B_{1}, A_{2}, B_{2} \in \mathfrak{A}:$ (4.13a) directly implies the counterpart of (4.11) for the $f_{A B}$. As for (4.11) itself, it follows from the combination of (4.13) and

$$
F_{A_{1} B_{1}}+\lambda \mathbb{1} \in \mathscr{D}_{T} \quad \text { with } \quad T\left(F_{A_{1} B_{1}}+\lambda \mathbb{1}\right)=G_{A_{1} B_{1}}+\lambda \mathbb{1}, \quad A_{1}, B_{1} \in \mathfrak{U},
$$

obtained from (4.12).

Our last criterion applies to states clustering of order 4 (without having to assume asymptotic abelianness).

Theorem 4.4. Let $\omega$, with the notation in Definition 3.2, be an $\alpha$-invariant state of a $C^{*}$-system $\{\mathfrak{U}, G, \alpha\}$, with $G$ abelian and $\mathfrak{A}$ unital. Let $\operatorname{Sp} U=\hat{G}$, and assume that $\omega$ is $\alpha$-clustering of order 4 . If there is a closable linear operator $T_{0}$ on $\mathscr{C}_{\infty}(G)$ (with its sup norm) such that $T_{0} f_{A B}=g_{A B}$ for all $A, B \in \mathfrak{A}, \omega$ is KMS for some continuous oneparameter subgroup of $G$.

Proof. We now use the $\mathscr{C}_{\infty}(G)$ version of Corollary 2.6, $T$ now denoting the closure of $T_{0}$ for the sup norm. The fact that $\mathscr{D}_{T}$ is dense in $\mathscr{C}_{\infty}(G)$ follows from Proposition 
3.4(iii). The proof that $T$ commutes with translations $\left(\mathscr{D}_{T}\right.$ being translation invariant), and that of the vanishing of $s_{0}$ are as above. And Remark 4.2 with $\mathscr{A}=\mathscr{C}_{\infty}(G), \mathscr{S}$ the linear span of $\mathscr{F}$, and $f_{1} \circ f_{2}=f_{1} f_{2}$ now applies to reduce the rest of the proof to showing that ${ }^{16}$

$$
f_{A_{1} A_{1}^{*}} \cdot f_{A_{2} A_{2}^{*}} \in \mathscr{D}_{T} \quad \text { with } \quad T\left(f_{A_{1} A_{1}^{*}} \cdot f_{A_{2} A_{2}^{*}}\right)=g_{A_{1} A_{1}^{*}} \cdot g_{A_{2} A_{2}^{*}}, \quad A_{1}, A_{2} \in \mathfrak{U},
$$

To establish this, we consider the following functions [analogous to the function in (3.28) but now chosen of positive type]

$$
\begin{aligned}
& \varphi_{u}^{\prime \prime}=f_{A_{1}^{\prime} \alpha_{u}\left(A_{2}^{\prime}\right), \alpha_{u}\left(A_{2}^{\prime *}\right) A_{1}^{*}} \\
& \psi_{u}^{\prime \prime}=g_{A_{1}^{\prime} \alpha_{u}\left(A_{2}^{\prime}\right), \alpha_{u}\left(A_{2}^{\prime *}\right) A_{1}^{\prime *}} \text { where }\left\{\begin{array}{l}
A_{1}^{\prime}=A_{1}-\omega\left(A_{1}\right) \mathbb{1} \\
A_{2}^{\prime}=A_{2}-\omega\left(A_{2}\right) \mathbb{1}
\end{array}, A_{1}, A_{2} \in \mathfrak{R}\right\}
\end{aligned}
$$

for which expanding in truncated functions now yields

$$
\left.\begin{array}{rl}
\varphi_{u}^{\prime \prime}(g)= & f_{A_{1} A_{1}^{*}} \cdot f_{A_{2} A_{2}^{*}}+\omega_{(4)}^{T}\left(\alpha_{u}\left(A_{2}^{*}\right), A_{1}^{*}, \alpha_{g}\left(A_{1}\right), \alpha_{g+u}\left(A_{2}\right)\right) \\
& +\omega_{(2)}^{T}\left(A_{1}^{*}, \alpha_{g+u}\left(A_{2}\right)\right) \omega_{(2)}^{T}\left(A_{2}^{*}, \alpha_{g-u}\left(A_{1}\right)\right) \\
\psi_{u}^{\prime \prime}(g)= & g_{A_{1} A_{1}^{*}} \cdot g_{A_{1} A_{2}^{*}}+\omega_{(4)}^{T}\left(\alpha_{g}\left(A_{1}\right), \alpha_{g+u}\left(A_{2}\right), \alpha_{u}\left(A_{2}^{*}\right), A_{1}^{*}\right) \\
& +\omega_{(2)}^{T}\left(\alpha_{g+u}\left(A_{2}\right), A_{1}^{*}\right) \omega_{(2)}^{T}\left(\alpha_{g-u}\left(A_{1}\right), A_{2}^{*}\right) .
\end{array}\right\}
$$

The fact that $\omega$ is $\alpha$-clustering of order 4 now implies that

$$
\left.\begin{array}{l}
\varphi_{u}^{\prime \prime} \underset{u=\infty}{\longrightarrow} f_{A_{1} A_{1}^{*}} \cdot f_{A_{2} A_{2}^{*}} \\
\psi_{u}^{\prime \prime} \underset{u=\infty}{\longrightarrow} g_{A_{1} A_{1}^{*}} \cdot g_{A_{2} A_{2}^{*}}
\end{array}\right\}
$$

in a manner dominated by a fixed $\mathscr{C}_{\infty}(G)$-function of $g$.

We now prove (4.14), whereby we can assume that neither $f_{A_{1} A_{1}^{*}}$ nor $f_{A_{2} A_{2}^{*}}$ vanishes since otherwise the result is trivial. Now (4.14) follows from (4.17) if the convergence there is uniform, which will be the case if it is uniform on compacts, since $\varphi_{u}^{\prime \prime}$ and $\psi_{u}^{\prime \prime}$ are $\mathscr{C}_{\infty}(G)$-dominated. Now simple convergence and domination imply convergence in the $\sigma\left(L^{\infty}(G), L^{1}(G)\right)$ topology by the dominated convergence theorem, and $\sigma\left(L^{\infty}(G), L^{1}(G)\right)$ convergence implies in turn convergence on compacts for positive type functions bounded above and below in sup norm. Thus it suffices to check that the functions $\varphi_{u}^{\prime \prime}$ and $\psi_{u}^{\prime \prime}$ are bounded below in norm by a positive constant. But this follows from simple convergence since the latter implies

$$
\left.\begin{array}{l}
\left\|\varphi_{u}^{\prime \prime}\right\|_{\infty}=\varphi_{u}^{\prime \prime}(0) \underset{u=\infty}{\longrightarrow}\left\|f_{A_{1} A_{1}^{*}}\right\|_{\infty} \cdot\left\|f_{A_{2} A_{2}^{*}}\right\|_{\infty} \\
\left\|\psi_{u}^{\prime \prime}\right\|_{\infty}=\psi_{u}(0) \underset{u=\infty}{\longrightarrow}\left\|g_{A_{1} A_{1}^{*}}\right\|_{\infty} \cdot\left\|g_{A_{2} A_{2}^{*}}\right\|_{\infty},
\end{array}\right\}
$$

where the first expression on the r.h.s. does not vanish by assumption, this holding also for the last from the existence of $T_{0}$.

We conclude with an alternative proof, along the lines of this paper, of a result first stated in [11] and first fully established in [9] ${ }^{17}$.

16 We used polarization to restrict yourselves to elements of $\mathfrak{f}^{+}$

17 The gap in [11], inherited from [12] and [7] consists in assuming the existence of a $\hat{g}_{A B}(E) \neq 0$ for all $E \in \mathbb{R}$ without motivating this assumption 
Theorem 4.5. Let $\{\mathfrak{A}, \mathbb{R}, \alpha\}$, with $\mathfrak{A}$ unital, be a $C^{*}$-system $L^{1}$-asymptotically abelian on a norm-dense, translation invariant $*$-subalgebra $\mathfrak{A}_{0}$ of $\mathfrak{A}$. Let $\omega$ be an $\alpha$-invariant state of $\mathfrak{A}$ and assume $\omega$ (i) stable for local perturbation of the dynamics ${ }^{18}$, (ii) $L^{1}$ clustering of order 4 on $\mathfrak{A}_{0}$. Then $\omega$ is either a ground or ceiling state (i.e. $\operatorname{Sp} U$ is one-sided) or it is $\beta$-KMS for some real $\beta$.

Proof. From stability one reaches as in [7], VI the "two-fold relation"

$$
f_{A_{1} B_{1}} \cdot f_{A_{2} B_{2}} d t=\int g_{A_{1} B_{1}} \cdot g_{A_{2} B_{2}} d t, \quad A_{1}, A_{2}, B_{1}, B_{2} \in \mathfrak{U}_{0} .
$$

Let then the net $A_{1}^{\sigma}, A_{2}^{\sigma}$ be such that

$$
\left.\begin{array}{l}
f_{A_{1}^{\sigma} B_{1}^{\sigma}} \underset{\sigma}{\longrightarrow} 0 \\
g_{A_{1} A_{1}^{\sigma} \underset{\sigma}{\longrightarrow}}^{\longrightarrow} g
\end{array}\right\}
$$

in $\sigma\left(L^{\infty}\left(\mathbb{R}, L^{1}(\mathbb{R})\right)\right.$. By dominated convergence we then have

$$
\int g g_{A_{2} B_{2}} d t=0, \quad A_{2}, B_{2} \in \mathfrak{A}_{0},
$$

which implies by Theorem 3.8 that $g=0$ since $\mathscr{F}_{0}$ if total in $B(\mathbb{R})$ by the normdensity of $\mathfrak{U}_{0}$ in $\mathfrak{H}$. The map $f_{A B} \rightarrow g_{A B}, A, B \in \mathfrak{U}_{0}$, thus fulfills the assumptions of Theorem 4.1 (see footnote there).

One could also, proceeding as in [7], VI, infer from (4.20) the relation

$$
\hat{f}_{A_{1} B_{1}}(E) \hat{g}_{A_{2} B_{2}}(E)=\hat{f}_{A_{2} B_{2}}(E) \hat{g}_{A_{1} B_{1}}(E), \quad E \in \mathbb{R},
$$

and use Theorem 3.8 to infer the existence of a continuous function $\Phi$ such that

$$
\hat{f}_{A B}(E)=\Phi(E) \hat{g}_{A B}(E), \quad E \in \mathbb{R},
$$

from which one immediately deduces the assumptions of Theorem 4.1 for the map $f_{A B} \rightarrow g_{A B}$.

\section{Appendix}

The following expansions are valid for arbitrary $A_{1}, A_{2}, B_{1}, B_{2}$ :

$$
\begin{aligned}
& f_{A_{1} \alpha_{u}\left(A_{2}\right), B_{1} \alpha_{u}\left(B_{2}\right)}(g)-f_{A_{1} B_{1}}(g) f_{A_{2} B_{2}}(g)-\omega\left(A_{1}\right) \omega\left(B_{1}\right) f_{A_{2} B_{2}}(g)-\omega\left(A_{2}\right) \omega\left(B_{2}\right) f_{A_{1} B_{1}}(g) \\
&=\omega\left(A_{1}\right) \omega\left(B_{2}\right) \omega_{(2)}^{T}\left(B_{1}, \alpha_{g+u}\left(A_{2}\right)\right)+\omega\left(A_{2}\right) \omega\left(B_{1}\right) \omega_{(2)}^{T}\left(B_{2}, \alpha_{g-u}\left(A_{1}\right)\right) \\
&+\omega_{(2)}^{T}\left(B_{1}, \alpha_{g+u}\left(A_{2}\right)\right) \omega_{(2)}^{T}\left(B_{2}, \alpha_{g-u}\left(A_{1}\right)\right)+\omega_{(4)}^{T}\left(B_{1}, \alpha_{u}\left(B_{2}\right), \alpha_{g}\left(A_{1}\right), \alpha_{g+u}\left(A_{2}\right)\right) \\
&+\omega\left(B_{1}\right) \omega_{(3)}^{T}\left(\alpha_{u}\left(B_{2}\right), \alpha_{g}\left(A_{1}\right), \alpha_{g+u}\left(A_{2}\right)\right)+\omega\left(B_{2}\right) \omega_{(3)}^{T}\left(B_{1}, \alpha_{g}\left(A_{1}\right), \alpha_{g+u}\left(A_{2}\right)\right) \\
&+\omega\left(A_{1}\right) \omega_{(3)}^{T}\left(B_{1}, \alpha_{u}\left(B_{2}\right), \alpha_{g+u}\left(A_{2}\right)\right)+\omega\left(A_{2}\right) \omega_{(3)}^{T}\left(B_{1}, \alpha_{u}\left(B_{2}\right), \alpha_{g}\left(A_{1}\right)\right), \\
& f_{A_{1} \alpha_{u}\left(A_{2}\right),\left[A_{1} \alpha_{u}\left(A_{2}\right)\right]^{*}}(g)-f_{A_{1} A_{1}^{*}}(g) f_{A_{2} A_{2}^{*}}(g)-\left|\omega\left(A_{1}\right)\right|^{2} f_{A_{2} A_{2}^{*}}-\left|\omega\left(A_{2}\right)\right|^{2} f_{A_{1} A_{1}^{*}} \overline{\omega\left(A_{1}\right)} \omega\left(A_{2}\right) \omega_{(2)}^{T}\left(A_{2}^{*}, \alpha_{g-u}\left(A_{1}\right)\right) \\
&=\left.\omega\left(A_{1}\right) \overline{\omega\left(A_{2}\right)} \omega_{(2)}^{T}\left(A_{1}^{*}, \alpha_{g+u}\left(A_{2}\right)\right)+\omega_{(4)}\right)\left(\alpha_{u}\left(A_{2}^{*}\right), A_{1}^{*}, \alpha_{g}\left(A_{1}\right), \alpha_{g+u}\left(A_{2}\right)\right) \\
&+\omega_{(2)}^{T}\left(A_{1}^{*}, \alpha_{g+u}\left(A_{2}\right)\right) \omega_{(2)}^{T}\left(A_{2}^{*}, \alpha_{g-u}\left(A_{1}\right)\right)+\omega_{(3)}^{T}\left(A_{1}\right) \\
&+\overline{\omega\left(A_{2}\right)} \omega_{(3)}^{T}\left(A_{1}^{*}, \alpha_{g}\left(A_{1}\right), \alpha_{g+u}\left(A_{2}\right)\right)+\overline{\omega\left(A_{1}\right)} \omega_{(3)}^{T}\left(\alpha_{u}\left(A_{2}^{*}\right), \alpha_{g}\left(A_{1}\right), \alpha_{g+u}\left(A_{2}\right)\right) \\
&+\omega\left(A_{1}\right) \omega_{(3)}^{T}\left(\alpha_{u}\left(A_{2}^{*}\right), A_{1}^{*}, \alpha_{g+u}\left(A_{2}\right)\right)+\omega\left(A_{2}\right) \omega_{(3)}^{T}\left(\alpha_{u}\left(A_{2}^{*}\right), A_{1}^{*}, \alpha_{g}\left(A_{1}\right)\right) .
\end{aligned}
$$

18 For the definition of this see [7], Definition 6.2 
The terms in the lines containing the equality signs are those motivating the introduction of the $A_{i}^{\prime}$ instead of the $A_{i}$ in the proofs of Proposition 3.8, resp. Theorem 4.4. $L^{1}$-clustering, resp. clustering, of order 4 makes the remaining terms tend to zero in a dominated manner as $u \rightarrow \infty$.

Acknowledgements. One of us (D. Kastler) acknowledges gratefully a useful discussion with Prof. J. Dixmier. The authors are indebted to Ministère des Universités, Paris, for the grant to one of them (M. Takesaki) of a guest professorship which made this collaboration possible.

\section{References}

1. Haag, R., Hugenholtz, N.M., Winnink, M.: On the equilibrium states in quantum statistical mechanics. Commun. Math. Phys. 5, 215-236 (1967)

2. Takesaki, M.: Tomita's theory of modular Hilbert algebras and its applications. Lecture notes in mathematics, 128. Berlin, Heidelberg, New York: Springer 1970

3. Araki, H., Haag, R., Kastler, D., Takesaki, M.: Extension of states and chemical potential. Commun. Math. Phys. 53, 97-134 (1977)

4. Tatsuuma, N.: An extension of AKHT theory of locally compact groups. Kokyuroku RIMS 314, $88(1977)$

5. Rudin, C.: Fourier analysis on groups. New York: Interscience 1962

6. Dixmier, J.: Les $C^{*}$-algèbres et leurs représentations. Paris : Gauthier-Villars 1964

7. Kastler, D.: Equilibrium states of matter and operator algebras. Symposia Math. 20, 49 (1976)

8. Takesaki, M., Tatsuuma, N.: Duality and subgroups. Ann. of Math. 93, 344 (1971)

9. Bratteli, O., Kishimoto, A., Robinson, D.W.: Stability properties and the KMS-condition. Commun. Math. Phys. 61, 209-238 (1978)

10. Hermann, R., Kastler, D.: Energy spectrum of extremal invariant states. Commun. Math. Phys. 56, 87-90 (1977)

11. Bratteli, O., Kastler, D. : Relaxing the clustering condition in the derivation of the KMS-property. Commun. Math. Phys. 46, 37-42 (1976)

12. Haag, R., Kastler, D., Trych-Pohlmeyer, E. : Stability and equilibrium. Commun. Math. Phys. 38, 173-193 (1974)

Communicated by H. Araki

Received March 20, 1979 